\title{
A CLASSICAL APPROACH TO EIGENVALUE PROBLEMS ASSOCIATED WITH A PAIR OF MIXED REGULAR STURM-LIOUVILLE EQUATIONS II
}

\author{
M. VENKATESULU and PALLAV KUMAR BARUAH \\ Sri Sathya Sai Institute of Higher Learning \\ Department of Mathematics and Computer Science \\ Prasanthinilayam 515134 \\ Andhra Pradesh, India
}

(Received February, 1995; Revised February, 2000)

\begin{abstract}
In the studies of acoustic waveguides in ocean, buckling of columns with variable cross sections in applied elasticity, transverse vibrations in nonhomogeneous strings, etc., we encounter a new class of problems of the type $L_{1} y_{1} \equiv-y_{1}^{\prime \prime}+q_{1}(x) y_{1}=\lambda y_{1} \quad$ defined on an interval $\left[d_{1}, d_{2}\right]$ and $L_{2} y_{2} \equiv-y_{2}^{\prime \prime}+q_{2}(x) y_{2}=\lambda y_{2}$ defined on the interval $\left[d_{2}, d_{3}\right]$ satisfying certain matching conditions at the interface point $x=d_{2}$.

Earlier, in Part I, we constructed a fundamental system for $\left(L_{1}, L_{2}\right)$ and derived certain estimates for the same.

Here, in Part II, we consider four types of boundary value problems associated with $\left(L_{1}, L_{2}\right)$ and study the corresponding spectra.

Key words: Sturm-Liouville Equations, Interface Boundary Conditions, Boundary Value Problems, Eigenfunctions, Spectrum.

AMS subject classifications: 34XX, 34B05, 34B10, 34B25.
\end{abstract}

\section{Introduction}

With the same notation as in Part I [4], consider the pair of Sturm-Liouville equations

$$
\begin{gathered}
L_{1} y_{1} \equiv-y_{1}^{\prime \prime}+q_{1}(x) y_{1}=\lambda y_{1}, \quad 0 \leq x \leq h, \\
L_{2} y_{2} \equiv-y_{2}^{\prime \prime}+q_{2}(x) y_{2}=\lambda y_{2}, \quad h \leq x \leq 1,
\end{gathered}
$$

together with the matching conditions at the interface $x=h$ given by

$$
y_{1}(h)=y_{2}(h), w_{1} y_{1}^{\prime}(h)=w_{2} y_{2}^{\prime}(h)
$$

where $\lambda$ is a complex constant, $0<h<1$ and $\left(q_{1}, q_{2}\right) \in L_{C}^{2}[0, h] \times L_{C}^{2}[h, 1]$.

Also consider the following set of boundary conditions at the end points $x=0$ and $x=1$.

Dirichlet boundary conditions: $\quad y_{1}(0)=y_{2}(1)=0$

Neumann boundary condition: $y_{1}^{\prime}(0)=y_{2}^{\prime}(1)=0$

Mixed boundary condition of Type A: $y_{1}(0)=y_{2}^{\prime}(1)=0$ 
Mixed boundary condition of Type B: $y_{1}^{\prime}(0)=y_{2}(1)=0$.

We call the boundary value problem (BVP) defined by (1)-(3) and (4) as BVP (I), the BVP defined by (1)-(3) and (5) as BVP (II), the BVP defined by (1)-(3) and (6) as BVP (III), the BVP defined by (1)-(3) and (7) as BVP (IV).

We say that the complex constant $\lambda$ is an eigenvalue of BVP (I), if BVP (I) has a nontrivial solution for that $\lambda$. The corresponding nontrivial solution(s) is (are) called the eigenfunction(s) for $\lambda$. The collection of all eigenvalues is called the spectrum of BVP (I). Similarly, we define the spectra of BVP (II, III, IV).

Note 1: It can be easily shown that th eigenvalues of BVP (I, II, III, IV) are identical with the zeros of the functions $y_{22}(1, \lambda), y_{12}^{\prime}(1, \lambda), y_{22}^{\prime}(1, \lambda), y_{12}(1, \lambda)$, respectively, where $\left(y_{11}, y_{12}\right),\left(y_{21}, y_{22}\right)$ are as in part I [4].

Note 2: Even if $w_{1}$ and $w_{2}$ are real with $\frac{w_{1}}{w_{2}}>0$, BVPs I-IV cannot be represented as selfadjoint operators acting on the product Hilbert space $L_{C}^{2}[0, h] \times L_{C}^{2}[h, 1]$ unless $w_{1}=w_{2}$.

Before presenting the main theorem, we state the following easily verified lemma.

Lemma 1: If $\left|z-\left(\frac{2 n+1}{2}\right) \pi\right| \geq \pi / 4$ for all integers $n$, then $\exp (|\operatorname{Im} z|) \leq|4 \cos z|$.

Assumption 1: We assume that $w_{1}, w_{2}$ are reals and that $\left|1-\frac{w_{1}}{w_{2}}\right|<1 / 4$.

Note 3: We note that Assumption 1 necessarily implies $\frac{w_{1}}{w_{2}}>0$.

Theorem 1: (Location of Eigenvalues) Let Assumption 1 be true.

(A) Let $N$ be an integer such that

$$
N>2\left(\frac{1+\left|1-\frac{w_{1}}{w_{2}}\right|}{1-4\left|1-\frac{w_{1}}{w_{2}}\right|}\right) \exp \left(\left(\left\|q_{1}\right\|^{2}+\left\|q_{2}\right\|^{2}\right)^{1 / 2}\right) \text {. }
$$

Then $y_{22}(1, \lambda)$ has exactly $N$ roots, counted with multiplicities, in the open half plane

$$
\operatorname{Re} \lambda<\left(N+\frac{1}{2}\right)^{2} \pi^{2}
$$

and for each $n>N$, exactly one simple root in th egged shaped region

$$
|\sqrt{\lambda}-n \pi|<\pi / 2 \text {. }
$$

There are no other roots.

(B) Let $N$ be an integer such that

$$
N>2\left(\frac{1+\left|1-\frac{w_{1}}{w_{2}}\right|}{1-4\left|1-\frac{w_{1}}{w_{2}}\right|}\right)\left(\left\|q_{1}\right\|^{2}+\left\|q_{2}\right\|^{2}\right)^{1 / 2} \exp \left(\left(\left\|q_{1}\right\|^{2}+\left\|q_{2}\right\|^{2}\right)^{1 / 2}\right)
$$

Then $y_{12}^{\prime}(1, \lambda)$ has exactly $N$ roots, counted with multiplicities, in the open half plane

$$
\operatorname{Re} \lambda<\left(N+\frac{1}{2}\right)^{2} \pi^{2}
$$

and for each $n>N$, exactly one simple root in the egged shaped region

There are no other roots.

$$
|\sqrt{\lambda}-n \pi|<\pi / 2 \text {. }
$$

$(C)$ Let $N$ be an integer satisfying inequality (8). Then $y_{22}^{\prime}(1, \lambda)$ has exactly $N$ roots, counted with multiplicities, in the open half plane

$$
\operatorname{Re} \lambda<N^{2} \pi^{2}
$$

and for each $n>N$, exactly one simple root in the egged shaped region 
$\sqrt{\lambda}-\left(\frac{2 n+1}{2}\right) \pi<\pi / 2$

There are no other roots.

$(D) \quad$ Let $N$ be an integer satisfying inequality $(9)$. Then $y_{12}(1, \lambda)$ has exactly $N$ roots, counted with multiplicities, in the open half plane

$$
\operatorname{Re} \lambda<N^{2} \pi^{2}
$$

and for each $n>N$, exactly one simple root in the egged shaped region

$$
\left|\sqrt{\lambda}-\left(\frac{2 n+1}{2}\right) \pi\right|<\pi / 2
$$

There are no other roots.

Proof: We prove only part $(A)$. Parts $(B),(C)$ and $(D)$ can be proven similarly.

(A) Consider a fixed integer $N$, and let $k>N$ be another integer. Consider the contours

$$
|\sqrt{\lambda}|+\left(k+\frac{1}{2}\right) \pi, \operatorname{Re} \sqrt{\lambda}=\left(N+\frac{1}{2}\right) \pi \text {, and }|\sqrt{\lambda}-N+1 \pi|=\pi / 2, n>N .
$$

For $\lambda$ belonging to the contours, it can be easily verified that the estimates

$$
\frac{2 N}{|\sqrt{\lambda}|}<1
$$

and by Lemma 1 (on p. 27 [3])

$$
\exp (|\operatorname{Im} \sqrt{\lambda}|)<4|\sin \sqrt{\lambda}|
$$

hold.

Let $N$ satisfy inequality (8). Then, for $\lambda$ belonging to each one of the above contours, by Theorem 3 (on p. 13 [3]), we get

$$
\begin{gathered}
\left|y_{22}(1, \lambda)-\frac{\sin \sqrt{\lambda}}{\sqrt{\lambda}}\right| \\
\leq \frac{\left|1-\frac{w_{1}}{w_{2}}\right|}{|\sqrt{\lambda}|} \exp (|\operatorname{Im} \sqrt{\lambda}|)+\frac{\left(1+\left|1-\frac{w_{1}}{w_{2}}\right|\right)}{|\lambda|} \exp \left(|\operatorname{Im} \sqrt{\lambda}|+\left(\left\|q_{1}\right\|^{2}+\left\|q_{1}\right\|^{2}\right)^{1 / 2}\right) \\
\leq\left[\left|1-\frac{w_{1}}{w_{2}}\right|+\frac{N\left(1-4\left|1-\frac{w_{1}}{w_{2}}\right|\right)}{2|\sqrt{\lambda}|}\right] \frac{\exp |\operatorname{Im} \sqrt{\lambda}|}{|\sqrt{\lambda}|} \text { (by (8)) } \\
<\left[4\left|1-\frac{w_{1}}{w_{2}}\right|+\frac{2 N}{|\sqrt{\lambda}|}\left(1-4\left|1-\frac{w_{1} \mid}{w_{2}}\right|\right)\right]\left|\frac{\sin \sqrt{\lambda}}{\sqrt{\lambda}}\right| \text { (by (11)) } \\
<\left|\frac{\sin \sqrt{\lambda}}{\sqrt{\lambda}}\right| \text { (by (10)). }
\end{gathered}
$$


Consequently, we get that $y_{22}(1, \lambda)$ does not vanish on the above contours. Also, we note that $y_{22}(1, \lambda)$ is an entire function of $\lambda$. Hence by Rouche's theorem ([2]), $y_{22}(1, \lambda)$ has as many roots, counted with multiplicities, as $\frac{\sin \sqrt{\lambda}}{\sqrt{\lambda}}$ in each of the above regions. Since $\sin \sqrt{\lambda} \sqrt{\lambda}$ has only the simple roots $n^{2} \pi^{2}, n \geq 1$ and since $k>N$ can be chosen arbitrarily large, the proof of part $(A)$ is complete.

The next theorem gives conditions under which the spectrum is real.

Theorem 2: Let $\left(q_{1}, q_{2}\right) \in L_{R}^{2}[0, h] \times L_{R}^{2}[h, 1]$ and assume $w_{1}, w_{2}$ are reals with $\frac{w_{1}}{w_{2}}>0$. Then the spectrum BVP $(I)$ is an infinite sequence of real numbers, which is bounded from below and tends to $+\infty$, for each $i=I, I I, I I I, I V$.

Proof: We prove the theorem for BVP (I). The proofs for the other BVPs can be given along similar lines.

Let $\lambda$ be an eigenvalue of BVP (I) and let $\left(y_{1}(\cdot, \lambda), y_{2}(\cdot, \lambda)\right)$ be a corresponding eigenfunction. Let $W\left[y_{i}(x, \lambda), \bar{y}_{i}(x, \lambda)\right]$ denote the Wronskian of $y_{i}$ and $\bar{y}_{i}$ for $i=1,2$. Then as in Theorem 1 (on p. 29 [3]), it can be shown that

$$
\begin{aligned}
& {\left[W\left[y_{1}(x, \lambda), \bar{y}_{1}(x, \lambda)\right]\right]^{\prime}=(\lambda-\bar{\lambda})\left|y_{1}(x, \lambda)\right|^{2}, \quad 0 \leq x \leq h} \\
& {\left[W\left[y_{2}(x, \lambda), \bar{y}_{2}(x, \lambda)\right]\right]^{\prime}=(\lambda-\bar{\lambda})\left|y_{2}(x, \lambda)\right|^{2}, \quad h \leq x \leq 1 .}
\end{aligned}
$$

Integrating the above relations with respect to $x$ on the respective intervals and using the Dirichlet boundary conditions, we get

$$
\begin{aligned}
& {\left[W\left[y_{1}(x, \lambda), \bar{y}_{1}(x, \lambda)\right]\right]_{x=h}=(\lambda-\bar{\lambda}) \int_{0}^{h}\left|y_{1}(x, \lambda)\right|^{2} d x} \\
& {\left[W\left[y_{2}(x, \lambda), \bar{y}_{2}(x, \lambda)\right]\right]_{x=h}=(\lambda-\bar{\lambda}) \int_{h}^{l}\left|y_{2}(x, \lambda)\right|{ }^{2} d x .}
\end{aligned}
$$

By using the matching conditions (3) satisfied by $\left(y_{1}(\cdot, \lambda), y_{2}(\cdot, \lambda)\right)$, Equations (12) and (13) yield

$$
(\lambda-\bar{\lambda})\left(\frac{w_{1}}{w_{2}} \int_{0}^{h}\left|y_{1}(x, \lambda)\right|^{2} d x+\int_{h}^{l}\left|y_{2}(x, \lambda)\right|^{2} d x\right)=0
$$

Since $\left(y_{1}, y_{2}\right)$ is a nontrivial pair and $\frac{w_{1}}{w_{2}}>0$, the above relation implies $\lambda=\bar{\lambda}$. Hence the spectrum of BVP (I) is real, and the rest follows from Theorem 1 (p. 29 [3]).

Finally, we present a theorem on the discreteness of the spectrum of BVPs (I)-(IV). Let $\dot{y}_{i i}(\cdot, \lambda)$ and $\dot{y}_{i j}^{\prime}(\cdot, \lambda)$ denote the derivatives of $y_{i j}(\cdot, \lambda)$ and $y_{i j}^{\prime}(\cdot, \lambda)$ with respect to $\lambda$, here $i, j=1,2$.

Theorem 3: Assume the hypotheses of Theorem 1 and let $w_{1}, w_{2}$ be reals with $\frac{w_{1}}{w_{2}}>0$.

(A) If $\lambda$ is an eigenvalue of $B V P(I)$, then

$$
\dot{y}_{22}(1, \lambda) y_{22}^{\prime}(1, \lambda)=\frac{w_{1}}{w_{2}} \int_{0}^{h}\left|y_{21}^{2}(x, \lambda)\right|^{2} d x+\int_{h}^{l}\left|y_{22}^{2}(x, \lambda)\right|^{2} d x .
$$

In particular, $\dot{y}_{22}(1, \lambda) \neq 0$ and (hence) all roots of $y_{22}(1, \lambda)$ are simple. 
(B) If $\lambda$ is an eigenvalue of BVP $(I I)$, then

$$
-y_{12}(1, \lambda) \dot{y}_{12}^{\prime}(1, \lambda)=\frac{w_{1}}{w_{2}} \int_{0}^{h}\left|y_{12}^{2}(x, \lambda)\right|^{2} d x+\int_{h}^{l}\left|y_{12}^{2}(x, \lambda)\right|^{2} d x .
$$

In particular, $\dot{y}_{12}^{\prime}(1, \lambda) \neq 0$ and (hence) all roots of $y_{12}^{\prime}(1, \lambda)$ are simple.

(C) If $\lambda$ is an eigenvalue of BVP (III), then

$$
-y_{22}(1, \lambda) \dot{y}_{22}^{\prime}(1, \lambda)=\frac{w_{1}}{w_{2}} \int_{0}^{h}\left|y_{21}^{2}(x, \lambda)\right|^{2} d x+\int_{h}^{l}\left|y_{22}^{2}(x, \lambda)\right|^{2} d x .
$$

In particular, $\dot{y}_{12}^{\prime}(1, \lambda) \neq 0$ and (hence) all roots of $y_{12}^{\prime}(1, \lambda)$ are simple.

(D) If $\lambda$ is an eigenvalue of $B V P(I V)$, then

$$
-\dot{y}_{12}(1, \lambda) y_{12}^{\prime}(1, \lambda)=\frac{w_{1}}{w_{2}} \int_{0}^{h}\left|y_{11}^{2}(x, \lambda)\right|^{2} d x+\int_{h}^{l}\left|y_{12}^{2}(x, \lambda)\right|^{2} d x .
$$

In particular, $y_{12}(1, \lambda) \neq 0$ and (hence) all roots of $y_{12}(a, \lambda)$ are simple.

Proof: We prove part $(A)$. The proofs of parts $(B)-(D)$ follow along similar lines.

$(A) \quad$ Let $\lambda$ be an eigenvalue of $\operatorname{BVP}(I)$. Then $\left(y_{21}(\cdot, \lambda), y_{22}(\cdot, \lambda)\right)$ satisfies Equations (1)-(3), the initial conditions

$$
y_{21}(0, \lambda)=0, y_{21}^{\prime}(0, \lambda)=1
$$

and the boundary conditions

$$
y_{22}(1, \lambda)=0 .
$$

Proceeding as in Theorem 2 (on p. 30 [3]), we get

$$
W\left[y_{21}(y, \lambda), \dot{y}_{21}(h, \lambda)\right]=-\int_{0}^{h} y_{21}^{2}(x, \lambda) d x
$$

and

$$
W\left[y_{22}(h, \lambda), \dot{y}_{22}(h, \lambda)\right]-\dot{y}_{22}(1, \lambda) y_{22}^{\prime}(1, \lambda)=-\int_{h}^{l} y_{22}^{2}(x, \lambda) d x .
$$

Multiplying Equation (14) by $\frac{w_{1}}{w_{2}}$ and adding this to Equation (15) of matching conditions at $x=h$, we get

$$
\begin{gathered}
\dot{y}_{22}(1, \lambda) y_{22}^{\prime}(1, \lambda)=\frac{w_{1}}{w_{2}} \int_{0}^{h} y_{21}^{2}(x, \lambda) d x+\int_{h}^{l} y_{22}^{2}(x, \lambda) d x \\
=\frac{w_{1}}{w_{2}} \int_{0}^{h}\left|y_{21}\right|^{2} d x+\int_{h}^{l}\left|y_{22}\right|^{2} d x
\end{gathered}
$$

since $y_{21}$ and $y_{22}$ are real-valued. Consequently, $\dot{y}_{22}(1, \lambda) \neq 0$ and $\lambda$ is a simple root of $y_{22}(1, \lambda)$. 


\section{Physical Application}

Acoustic wave guides in oceans [1]: The following problem is encountered in the study of 'acoustic wave guides in oceans' when the ocean is considered to consist of two layers:

$$
\begin{aligned}
& -\left[z_{1}^{\prime \prime}(t)+k_{1}^{2} z_{1}(t)\right]=\lambda z_{1}(t), 0 \leq t \leq d_{1}, \\
& =\left[z_{2}^{\prime \prime}(t)+k_{21}^{2} z_{2}(t)\right]=\lambda z_{2}(t), d_{1} \leq t \leq d_{2},
\end{aligned}
$$

together with mixed boundary conditions given by

$$
\begin{gathered}
z_{1}\left(d_{1}\right)=z_{2}\left(d_{1}\right), \frac{1}{\rho_{1}} z_{1}^{\prime}\left(d_{1}\right)=\frac{1}{\rho_{2}} z_{2}^{\prime}\left(d_{1}\right) \\
z_{1}(0)=z_{2}^{\prime}\left(d_{2}\right)=0
\end{gathered}
$$

where $\rho_{1}, \rho_{2}$ are (positive) constant densities of the two layers; $k_{1}, k_{2}$ are the constants which depend upon the frequency constant $w$ and constant sound velocities $c_{1}, c_{2}$ of the two layers, $\lambda$ is an unknown constant (eigenvalue), $\left[0, d_{1}\right]$ and $\left[d_{1}, d_{2}\right]$ denote the two layers and $z_{1}, z_{2}$

denote the depth eigenfunctions. From the physics of the problem, it follows that the absolute difference between the densities $\rho_{1}, \rho_{2}$ is relatively very small, and therefore we may assume that

$$
\left|1-\frac{\rho_{2}}{\rho_{1}}\right|<1 / 4
$$

Under the transformation $x=t / d_{2}$, BVP (16)-(19) can be shown to be of the type BVP III. Now, choose $N$ such that

$$
\begin{gathered}
N>2\left(\frac{1+\left|1-\frac{\rho_{2}}{\rho_{1}}\right|}{1-4\left|1-\frac{\rho_{2}}{\rho_{1}}\right|}\right) d_{2}\left(k_{1}^{4} d_{2} d_{1}+k_{2}^{4} d_{2}\left(d_{2}-d_{1}\right)\right)^{1 / 2} \\
\quad \times \exp \left(d_{2}\left(k_{1}^{4} d_{2} d_{1}+k_{2}^{4} d_{2}\left(d_{2}-d_{1}\right)\right)^{1 / 2}\right) .
\end{gathered}
$$

Then applying Theorem $1(C)$, it can be shown that the problem (16)-(19) has

exactly $N$ eigenvalues in the open half plane $\operatorname{Re} \lambda<\frac{N^{2} \pi^{2}}{d_{2}^{2}}$, and for each $n>N$, exactly one eigenvalue in the egg-shaped region

$$
\left|\sqrt{\lambda}-\left(\frac{2 n+1}{2}\right) \frac{\pi}{d_{2}}\right|<\frac{\pi}{2 d_{2}} .
$$

Also, by Theorems $3(C)$ and 2, these eigenvalues are simple and form an infinite sequence of real numbers, which is bounded from below and tends to $+\infty$.

\section{Acknowledgement}

The authors thank the referee for the useful suggestions and comments which resulted in the present form of the paper. The authors dedicate the work to the Chancellor of the Institute Bhagawan Sri Satya Sai Baba. 


\section{References}

[1] Boyles, C.A., Acoustic Waveguides, Applications to Oceanic Sciences, Wiley, New York 1984.

[2] Boas, R.P., Introduction to Complex Analysis, Random House, New York 1987.

[3] Poschel, J. and Trubowitz, E., Inverse Spectral Theory, Academic Press, New York 1987.

[4] Venkatesulu, M. and Baruah, P.K., A classical approach to eigenvalue problems associated with a pair of mixed regular Sturm-Liouville equations I, JAMSA, 14:2 (2001), 205-214. 


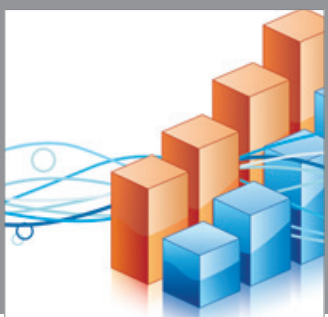

Advances in

Operations Research

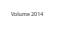

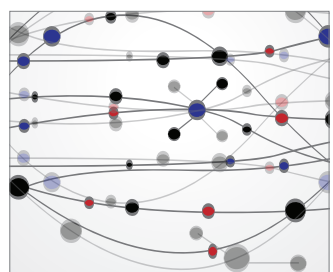

\section{The Scientific} World Journal
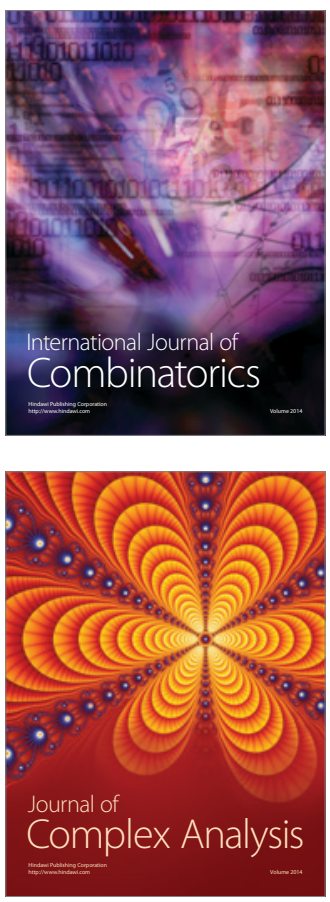

International Journal of

Mathematics and

Mathematical

Sciences
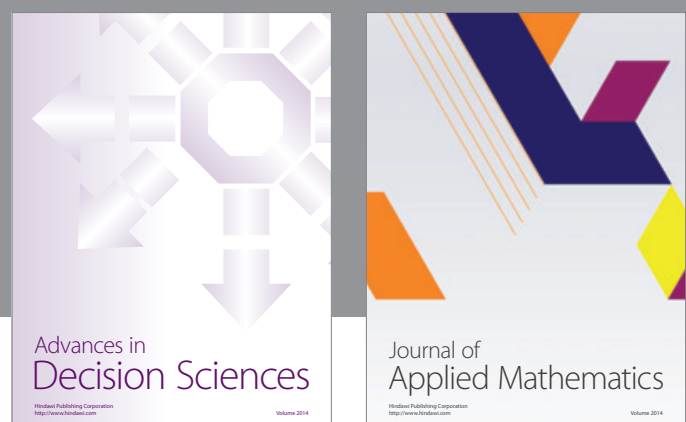

Journal of

Applied Mathematics
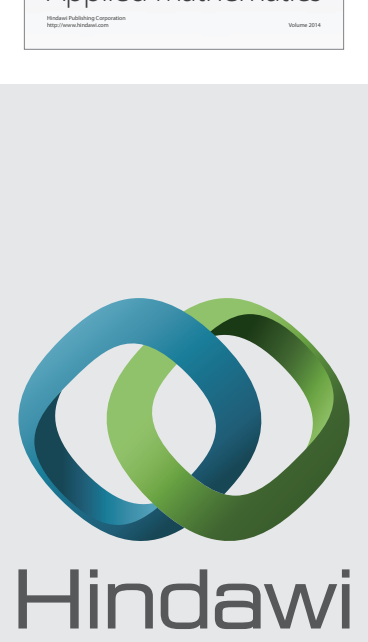

Submit your manuscripts at http://www.hindawi.com
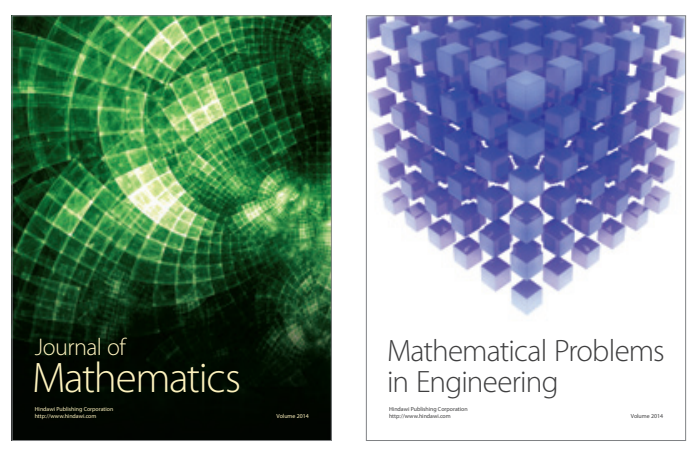

Mathematical Problems in Engineering
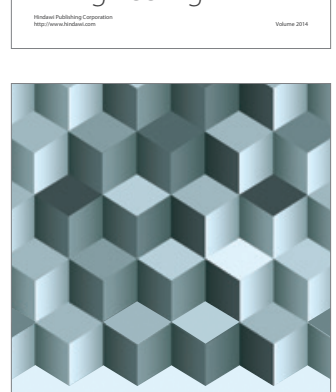

Journal of

Function Spaces
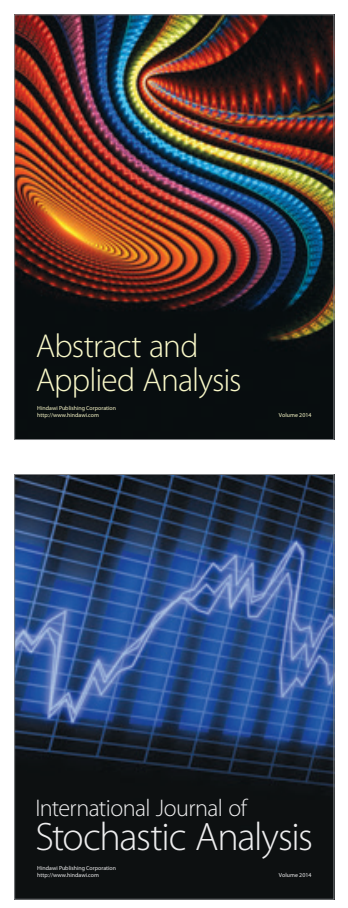

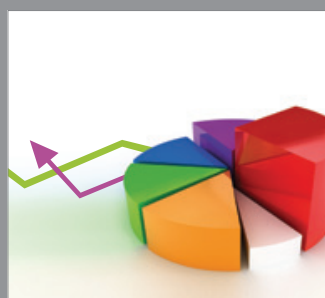

ournal of

Probability and Statistics

Promensencen
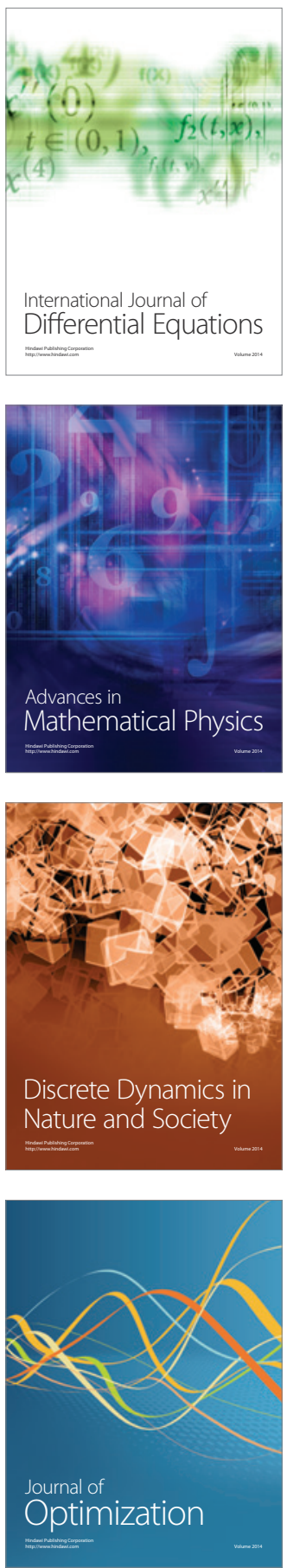